\title{
SIMULATIONS OF THE ROTATING POSITRON TARGET IN THE PRESENCE OF OMD FIELD
}

\author{
S. Antipov, L. K. Spentzouris, IL Inst. of Technology, Chicago IL, 60616, U.S.A. \\ W. Gai, W.Liu, ANL, Argonne, IL, 60439, U.S.A.
}

\begin{abstract}
For an International Linear Collider (ILC) undulatorbased positron source target configuration, a strong optical matching device (OMD) field is needed inside the target to increase the positron yield (by more than 40\%) [1]. It is also required that the positron target is constantly rotated to reduce thermal and radiation damage. We report on a simulation of the rotating metal target wheel under a strong magnetic field. By rearranging Maxwell's equations for a rotating frame and using Comsol [5], we have solved the detailed magnetic field distribution and eddy current of a rotating metal disk in magnetic field, and so the required power to drive the target wheel. In order to validate the simulation process, we have compared our results with previous experimental data [2] and found they are in very good agreement. Here we give detailed results on the proposed ILC target system, such as induced magnetic field (dipole and higher orders), eddy current distribution and the driving force requirements. The effect of these higher order fields on the positron beam dynamics is also considered.
\end{abstract}

\section{INTRODUCTION}

The problem of the ILC positron target rotation in a strong magnetic field produced by an optical matching device (OMD) is similar to the magnetic brake. A simple empirical scaling model was developed [2,3], and later refined by Qian [4]. But these models only fit the low velocity region and produce large errors in the region of high rotational speed.

We formulate the problem as follows: solve for Eddy currents induced in a conducting disk (target) spinning in the presence of external magnetic field (OMD). So far, the OMDs under the consideration (either superconducting or normal) have constant magnetic fields. Our focus will be on studies and simulations of a conducting disk rotating in a constant magnetic field. This problem does not have an exact solution.

To simulate this problem we directly include the fact that the target rotates in Maxwell's equations and solve the modified equations. This approach brings us to a nonstandard equation for induced magnetic field [8]:

$$
\nabla^{2} \vec{B}+\sigma \cdot \mu \cdot \nabla \times\left(\vec{v} \times\left(\vec{B}+\vec{B}_{0}\right)\right)=0
$$

Here $B$ is the magnetic field produced by eddy currents, $\nabla \times \vec{B}=\mu \vec{j} ; B_{0}$ is the external magnetic field, $\sigma$ is the disc conductivity and $\vec{v}=\omega \cdot\{-y, x, 0\}$ is a velocity vector field at the point $(x, y, z)$ on the disk. We consider the disk spinning in the $X Y$ plane.

We used the COMSOL (FEMLAB) - finite element method software to simulate this problem [5]. The finite element method allows effective mesh configurations. In our case, the Eddy currents are excited in the proximity of the applied magnetic field. Therefore, we use a dense mesh in the area of interest, and a less dense mesh in the remaining subdomains (figure 1).

\section{GEOMETRIES STUDIED}

Once the physics of the model was set we simulated several different geometries of the target and magnet system [8].

\section{LLNL / SLAC Experiment}

A group of LLNL/SLAC scientists [6] conducted a series of experiments with copper and aluminum disks with diameters of 9 and 10 inches, and thicknesses of 0.9 and 1 inch respectively. The disk was rotated in the magnetic field produced by a single pole magnet of $1 \mathrm{~cm}$ aperture. The distance between the magnet and the disk was $0.1,0.05$ or $0.01 \mathrm{inch}$. The force acting on the magnet from the spinning disk was measured using strain gauges. This force is equal to the drag force. During the experiment the roll off of the force at higher frequencies of target rotation that was expected from the approximate models was not observed.

We simulated the exact geometry of the experiment. Our simulation was in perfect agreement with the experimental data (drag force as a function of the target rotation frequency) [8]. LLNL has also performed the simulation of their experiment [7]. Due to high memory requirements their method was limited to low frequencies of target rotation.

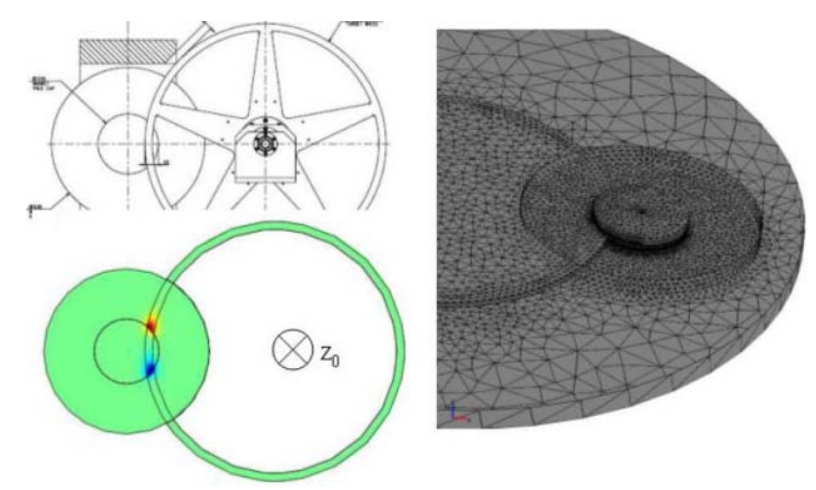

Figure 1: Typical simulation geometry. Technical drawing of Cockroft Institute prototype target. Simulation: geometry and induced magnetic field (z) at 3000rpm. Mesh is enhanced near the area of interest. 


\section{ILC Prototype Experiment}

The prototype experiment is planned to be conducted in Cockcroft Institute, Daresbury Laboratory, UK. Our simulation is used to determine power requirements to keep the target spinning. To reduce the volume of conductor moving in the presence of magnetic field the ILC target is a rim instead of solid disk.

The prototype target is twice as small $(1 \mathrm{~m})$ in diameter as proposed ILC target. Therefore the rotational frequency of interest is twice as large (2000rpm) to maintain the same linear velocity of the rim. Rim size $30 \mathrm{~mm}, 14 \mathrm{~mm}$ thickness. The target is placed between the magnet poles (figure 1) of $25 \mathrm{~cm}$ diameter. We pick 1.5 Tesla constant field on the target for simulation. The distance between magnet poles is $5 \mathrm{~cm}$. Therefore magnetic field stays almost across the target (drops to 1.497 Tesla).

We perform parametric simulation with target rotational frequency and target conductivity as parameters. Results for stopping (drag) force are presented on figure 2 .

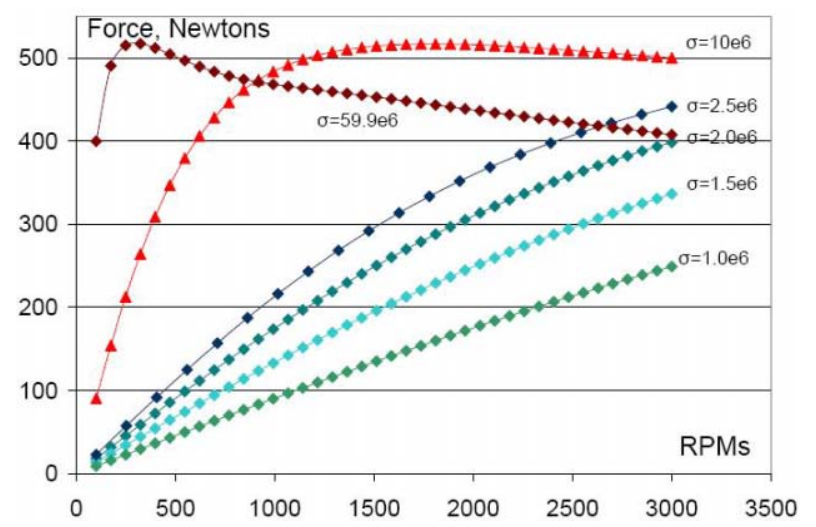

Figure 2: Stopping force (Newtons) as a function of rotational frequency for different conductivities. For neartitanium conductivity there is no roll-off prior to 3000RPM. For comparison we show, that copper target will have a roll-off near $400 R P M \mathrm{~s}$.

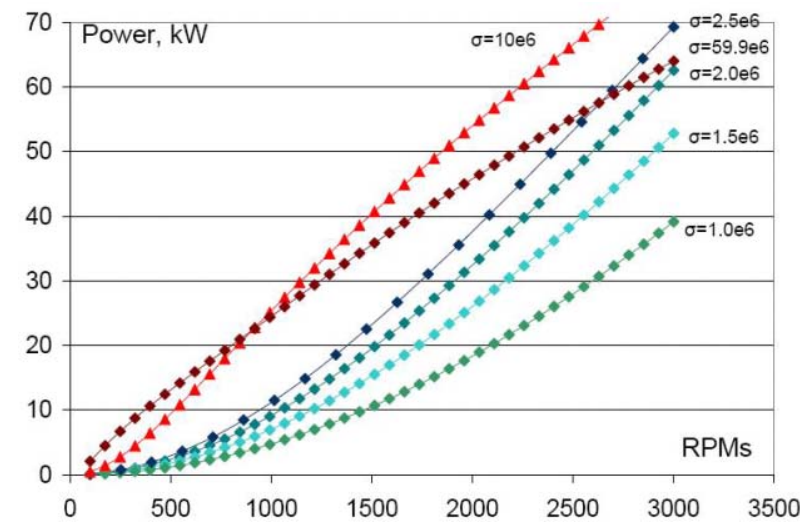

Figure 3: Power requirements depending on material conductivity as a function of RPMs, for $0.5 \mathrm{~m}$ radii disk.

Drag force determines the power requirement to keep the prototype target spinning. The power is dominated by the frequency of rotation term. The results are on figure 3. As expected, materials with lower value of conductivity require less power to be moved in magnetic field.

\section{Full-Scale ILC Geometry}

The ILC positron target is a $2 \mathrm{~m}$ diameter, $1.4 \mathrm{~cm}$ thick conducting disk. It rotates in the optical matching device (OMD) magnetic field. The magnetic field reaches the value of 5 Tesla inside the disk. We use our method to simulate this geometry.

We performed extensive parametric simulations of the target with frequency of rotation, conductivity of the target and geometry as parameters [8]. The ILC target is intended to operate at 1000 RPM rotation rate [9]. Our results show that a solid disk configuration requires about $800 \mathrm{kWatts}$ to keep the target spinning. Therefore we consider a ring configuration. Varying the ring width and magnet aperture we achieved a much more realistic number of $170 \mathrm{~kW}$ for $\sigma=1.5 \cdot 10^{6}$ (titanium alloy) and 1.5 $\mathrm{cm}$ ring width.

Another important effect associated with the target rotation is attenuation of the total field inside the target. The field from the optical matching device (OMD), $\mathrm{B}_{0}$, induces the eddy currents. The eddy currents, in turn produce the field, B. According to Lenz's law this field is trying to reduce the external field. This effect depends strongly on the rotational frequency. Figure 4 (right) shows the major component $(\mathrm{z})$ of the total field $\left(\mathrm{B}+\mathrm{B}_{0}\right)$ as a function of $\mathrm{z}$ at different rotational frequencies for a $3 \mathrm{~cm}$ wide target with a $3 \mathrm{~cm}$ aperture magnet with external field 5 Tesla. The faster the target rotates, the lower the field. At design frequency of 1000 RPMs, the total field is 2.7 Tesla. The field restores quickly back to 5 Tesla, therefore the positron yield does not suffer.
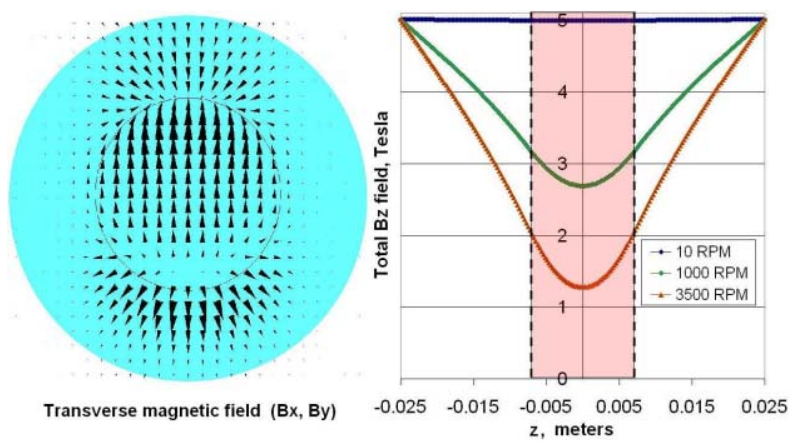

Figure 4: Left: Deflecting magnetic field $\left(\mathrm{B}_{\mathrm{x}}, \mathrm{B}_{\mathrm{y}}\right)$. Ring in the center is a beampipe. Right: Total (external and induced) z-component of magnetic field at 10, 1000 (design) and 3500 RPM. Shaded area is the target.

The transverse components of the induced field are another issue for target development. They create the deflection field. Figure 4 (left) presents $\mathrm{x}$ and $\mathrm{y}$ components of the induced magnetic field $5 \mathrm{~mm}$ away from the surface of the target. This is a first order of magnitude effect ( $\sim 0.2$ Tesla) [8]. As can be seen on Figure 4 (left) it is $\mathrm{B}_{\mathrm{y}}$ field that will deflect the beam. 
Particles with low energies will be steered more dramatically. The lowest captured energy of the positron from the target is $3 \mathrm{MeV}$. Further we simply integrate the equation of motion with the Lorentz force, produced by a $\mathrm{B}_{\mathrm{y}}$ component of magnetic field along the particle path. The maximum deflection neglecting the focusing properties of the solenoid in this case is $3 \mathrm{~mm}$ on the length where $\mathrm{B}_{\mathrm{y}}$ is still significant. A correction system can be designed based on the simulation results to steer the beam back. Since the deflection is dominated by a single component of magnetic field a simple dipole magnet can do the job.

The detailed analysis (figure 5) shows, that for most cases the correction system is not required. The deflecting field kicks some particles out, but it also kicks other ones in. In some cases due to the broad band matching provided by the OMD field, the distortion of field does not cause any noticeable change to the e+ yield. In cases when conductivity of the target is higher than $3 \cdot 10^{6}$ the loss of yield is about $5 \%$.

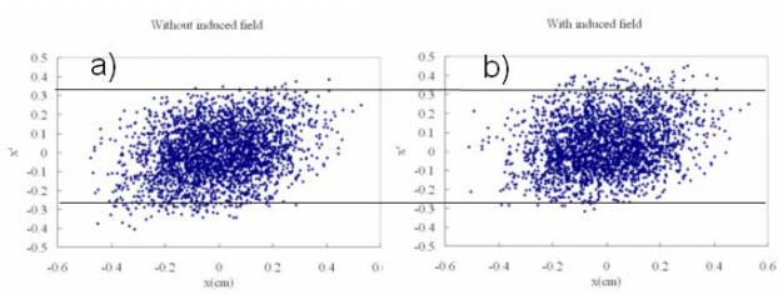

Figure 5: Initial $x-x$ ' phase space of captured positrons. a) without induced field. b) with induced field. The loss of yield is less than $5 \%$.

\section{SUMMARY}

We have developed a model that numerically solves the problem of a conducting disk spinning in a magnetic field. The simulation was checked against the SLAC/LLNL experiment. Very good agreement is reported. We have performed general studies for the ILC positron target and its prototype. Parametric studies were done. These simulations help to determine a realistic design for the ILC positron target: OMD aperture, thickness of the rim in a ring configuration of the target and conductivity of the target.

We discussed several effects associated with the target rotation and their effect on the beam. Reduction of OMD field by the induced field can be made less dramatic at lower rotational frequencies. The deflection field induced by rotation can be simulated. Based on the simulation a correction system can be developed if needed.

The submitted paper has been created by UChicago Argonne, LLC, Operator of Argonne National Laboratory ("Argonne"). Argonne, a U.S. Department of Energy Office of Science laboratory, is operated under Contract No. DE-AC02-06CH11357.
This work is also supported by the National Science Foundation grant \# 0237162.

\section{REFERENCES}

[1] Y.K.Batygin. "Positron Collection in Linear Collider" Proceedings of 2005 ALCPG \& ILC Workshops - Snowmass, U.S.A., August 14-27, 2005.

[2] J.H. Wouterse. "Critical Torque and speed of eddy current Brake with widely separated soft iron poles" IEEE Proceedings-B Vol. 138, No.4, July 1991, pp153-158

[3] W. R. Smythe. "On Eddy Currents in a Rotating Disk" Trans. AIEE, 1942, 61, pp. 681-684

[4] P. Kachroo, M. Qian. "Modeling and Control of Electromagnetic Brakes for Enhanced Braking Capabilities for Automated Highway Systems" IEEE Conference on Intelligent Transportation Systems ITSC'97, pp. 391-396, 1997.

[5] COMSOL Multiphysics user guide (www.comsol.com)

[6] W. T. Piggott, S. Walston, D. Mayhall "Preliminary Investigations of Eddy Current Effects on a Spinning Disk." LLNL report. Sept. 2006.

[7] D. J. Mayhall, W. Stein, J. B. Gronberg "Computer Calculations of Eddy-Current Power Loss in Rotating Titanium Wheels and Rims in Localized Axial Magnetic Fields" UCRL-TR-221440.

[8] Numerical studies of ILC positron target and OMD field effects on beam. S. Antipov, W. Liu, W.Gai, L. Spentzouris. In press. Jour. Appl. Phys. JR07-0632R.

[9] ILC Global Design Effort: Summary of WG3a. Proceedings of 2005 ALCPG \& ILC Workshops Snowmass, U.S.A., August 14-27, 2005. 